\title{
Cardiac fibrosis research: two steps forward
}

This novel
induction
protocol
for human
iPSC-derived
cardiac
fibroblasts
will enable
the study of
sex-specific and
patient-specific
cellular
responses

Resident cardiac fibroblasts are the main cell type contributing to cardiac fibrosis, but their properties and activation dynamics are poorly understood. Now, two studies reporting novel insights into fibroblast identity and dynamics provide important steps forward in our understanding of cardiac fibrosis and the quest to develop new anti-fibrotic strategies.

Rackham, Cook and colleagues report the first genome-wide data set on protein translation dynamics during cardiac fibroblast activation, describe the mRNA transcripts that undergo translation during the fibroblast transition to myofibroblast in response to transforming growth factor- $\beta 1$ (TGF $\beta 1)$ and identify a network of post-transcriptional regulators. "Being able to disentangle transcriptional and translational signatures elicited by TGF $\beta 1$ is important not only to evaluate therapeutic interventions targeting TGF $\beta 1$, but also to unveil novel molecular pathways regulating disease onset and pathophysiology," says Rackham.

RNA sequencing (RNA-seq) and

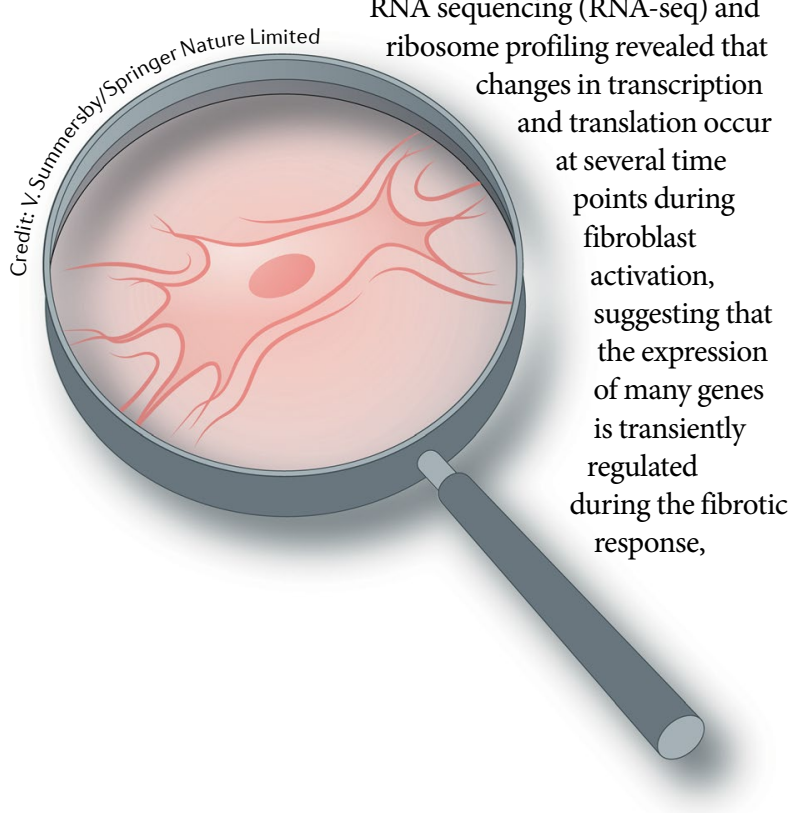

and approximately one-third of the expressed genes underwent translational regulation. Targets of RNA-binding proteins were strongly enriched among post-transcriptionally regulated genes. Analysis of ribosome occupancy in heart samples from 30 patients with dilated cardiomyopathy indicated

that these post-transcriptional regulatory networks are also active in the fibrotic heart.

"The results of Rackham, Cook and colleagues are particularly exciting for two reasons. First, they show a more valid means of assessing gene expression in cells during disease or transition states, and second, they identify a number of interesting novel gene candidates as fibrotic mediators that would have otherwise been missed," comments Jeffery Molkentin (Cincinnati Children's Hospital Medical Center, USA), who was not involved in either study.

In a separate study, Wu and colleagues performed an in-depth analysis of a previously published mouse transcriptome atlas generated by single-cell RNA-seq - to define the transcriptome profiles of different tissue-specific fibroblasts. The analysis showed that all fibroblasts express a common set of fibroblast-specific genes but each tissue-specific fibroblast also has a unique gene expression signature, revealing that fibroblast identity is distinguished, and probably determined, by a small number of transcription factors. Transcriptome signatures were conserved in human primary fibroblasts. For cardiac fibroblasts in particular, the analyses indicated that these cells are mostly of the epicardial lineage and, on the basis of this finding, the researchers developed a protocol to generate cardiac fibroblasts from human induced pluripotent stem cells
(iPSCs). The iPSC-derived cardiac fibroblasts had a quiescent phenotype, recapitulated most of the fundamental characteristics of primary cardiac fibroblasts and were sensitive to pro-fibrotic and anti-fibrotic agents. Using this platform, complementary receptor-ligand interaction analysis showed that cardiac fibroblasts can interact with many cardiac cell types via different signalling pathways. For example, crosstalk between atrial natriuretic peptide and/or B-type natriuretic peptide in cardiomyocytes and natriuretic peptide receptor 1 in cardiac fibroblasts is involved in suppressing fibrosis.

"This novel induction protocol for human iPSC-derived cardiac fibroblasts will enable the study of sex-specific and patient-specific cellular responses for fibrosis mechanistic studies in vitro," comments Wu. "Given that human iPSCs provide an unlimited cell source to generate a large quantity of cardiac fibroblasts, this platform can prove valuable for high-throughput screening of anti-fibrotic and pro-fibrotic drugs," he adds.

"These two studies fill a large hole in the literature and experimental repertoire of the cardiac fibroblast so that the field can now progress much further in our understanding of these cells and their function, and how we might control their activity for novel drug development and therapeutic purposes," concludes Molkentin.

Irene Fernández-Ruiz ORIGINAL ARTICLES Zhang, $\mathrm{H}$. et al. Generation of quiescent cardiac fibroblasts from human induced pluripotent stem cells for in vitro modeling of cardiac fibrosis. Circ. Res. https:// doi.org/10.1161/CIRCRESAHA.119.315491 (2019) | Chothani, S. et al. Widespread translational control of fibrosis in the human heart by RNAbinding proteins. Circulation https://doi.org/ 10.1161/CIRCULATIONAHA.119.039596 (2019) FURTHER READING Tallquist, M. D. \& Molkentin, J.D. et al. Redefining the identity of cardiac fibroblasts. Nat. Rev. Cardiol. 14, 484-491 (2017) 